\title{
P1-082
}

\section{Application of the radiating divertor approach to innovative tokamak divertor concepts}

\author{
T.W. Petrie ${ }^{a^{*}}$, S.L. Allen ${ }^{b}$, M.E. Fenstermacher ${ }^{b}$, R.J. Groebner ${ }^{a}$, C.T. Holcomb ${ }^{b}$, \\ E. Kolemen ${ }^{c}$, R.J. La Haye ${ }^{a}$, C.J. Lasnier ${ }^{b}$, A.W. Leonard ${ }^{a}$, T.C. Luce ${ }^{a}$, A.G. McLean ${ }^{b}$, \\ R. Maingi ${ }^{\text {C }}$, R.A. Moyer ${ }^{\text {D }}$, W.M. Solomon ${ }^{\text {C }}$, V.A. Soukhanovskii ${ }^{\text {, }}$ F. Turco ${ }^{\text {e, }}$, \\ and J.G. Watkins ${ }^{\text {f }}$ \\ ${ }^{a}$ General Atomics, PO Box 85608, San Diego, California 92186-5608, USA \\ ${ }^{b}$ Lawrence Livermore National Laboratory, 700 East Ave, Livermore, California 94550, USA \\ ${ }^{c}$ Princeton Plasma Physics Laboratory, PO Box 451, Princeton, New Jersey 08543-0451, USA \\ ${ }^{d}$ University of California San Diego, 9500 Gilman Dr., La Jolla, California 92093-0417, USA \\ ${ }^{e}$ Columbia University, 2960 Broadway, New York, New York 10027, USA \\ ${ }^{f}$ Sandia National Laboratory, PO Box 5800, Albuquerque, New Mexico 87185, USA
}

\begin{abstract}
We survey the results of recent DIII-D experiments that tested the effectiveness of three innovative tokamak divertor concepts in reducing divertor heat flux while still maintaining acceptable energy confinement under neon/deuterium-based radiating divertor (RD) conditions: (1) magnetically unbalanced high performance double-null divertor (DND) plasmas, (2) high performance doublenull "Snowflake" (SF-DN) plasmas, and (3) single-null H-mode plasmas having different isolation from their divertor targets. In general, all three concepts adapt well to RD conditions, achieving significant reduction in divertor heat flux $\left(q_{\perp p}\right)$ and maintaining high performance metrics, e.g., $50 \%-70 \%$ reduction in peak divertor heat flux for DND and SF-DN plasmas that are characterized by $\beta_{\mathrm{N}} \cong 3.0$ and $H_{98(\mathrm{y}, 2)} \approx 1.35$. It is also demonstrated that $q_{\perp p}$ could be reduced $\approx 50 \%$ by extending the parallel connection length $\left(L_{\mid-\mathrm{XPT}}\right)$ in the scrape-off layer between the X-point and divertor targets over a variety of the RD and non-RD environments tested.

Abstract length (150 words): 148 currently

PACS: $52.25 \mathrm{Vy}, 52.55 . \mathrm{Fa}, 52.55 . \mathrm{Rk}, 89.30 . \mathrm{Jj}$

JNM keywords: Divertor Materials (D0500), Impurities (I0100), Plasma-Materials Interaction (P0500), Plasma Properties (P0600)

PSI-21 keywords: DIII-D, Divertor geometry, Impurity seeding, Neon, Power deposition

*Corresponding and presenting author address: General Atomics, PO Box 85608, San Diego, California 92186-5608, USA

*Corresponding and presenting author e-mail: petrie@,fusion.gat.com
\end{abstract}




\section{Introduction}

Future high-powered tokamaks will need a way to achieve acceptable steady power loading at the divertor targets. Previous investigations have shown that significant reductions in heat loading are possible by enhancing radiated power through a combination of deuterium and/or impurity injection, e.g., "puff-and-pump" [1-4]. In addition, by configuring the divertor(s) in ways to exploit physics that are favorable to heat flux reduction (e.g., lengthening the parallel connection length of the divertor legs), further reductions in divertor heating, in principle, may be possible. In this paper, we make preliminary assessments of recent DIII-D experiments that tested the efficacy of combining three such divertor design concepts with the "puff-and-pump" radiating divertor approach. These include: (1) high performance standard double-null divertor (DND) plasmas [Fig. 1(a)], (2) high performance double-null "Snowflake" (SF-DN) plasmas [5] [Fig. 1(b)], and (3) single-null H-mode plasmas with different isolation from their divertor targets [6] [Fig. 1(c,d)].

The DND shape is often considered as the model configuration for "advanced" high performance tokamak plasmas because of the advantages in confinement and stability offered by high triangularity [7,8]. Previously on DIII-D, the "puff-and-pump" radiating divertor approach using argon as the seed impurity was applied to DND H-mode plasmas and produced significant reduction in divertor heating [4]. For the DND plasmas discussed in this paper in Sec. 3.1, we focus on the response of high performance DND plasmas, i.e., those with normalized plasma beta $\beta_{\mathrm{N}} \approx 3$ and energy confinement time $H_{98(\mathrm{y}, 2)} \approx 1.35$, to radiating divertor conditions using neon as the seed impurity.

The Snowflake divertor has been shown to be effective in dissipating heat flux at the divertor target for single-null discharges in both NSTX [9] and DIII-D [10]. The Snowflake divertor used in this study is configured by adding a second X-point near the outer divertor target (i.e., "Snowflake- 
minus" [5]). As a result, the poloidal flux expansion around the outer divertor target $\left(F_{\mathrm{XP}-\mathrm{OD}}\right)$ is significantly increased and the incoming power flow to the outer divertor is more evenly dispersed compared with a more conventional divertor configuration that does not have the second X-point. In this paper, we compare plasma performance of the Snowflake under operating conditions that are similar to the DND above [Sec. 3(b)]. As with the DND, we choose the high triangularity doublenull (DN-SF) configuration.

Previous studies on DIII-D showed that by extending the parallel connection length between the X-point and the outer divertor target $\left(L_{\mid-\mathrm{XPT}}\right)$ of single-null H-mode plasmas produced broader heat flux profiles at the outer divertor target and lower peak heat fluxes, despite higher poloidal flux expansion $\left(F_{\mathrm{XP}-\mathrm{OD}}\right)$ at shorter $L_{\mid \vdash \mathrm{XPT}}[11]$. SOLPS modeling [12] of those data indicated that cross-field transport was an important consideration and that perpendicular transport naturally would gain in importance relative to parallel transport as $L_{\mid \vdash \mathrm{XPT}}$ was extended. In Sec. 3(c), we reexamine whether the advantages of longer $L_{\mid-\mathrm{XPT}}$ on reduced heat flux are maintained when these same plasmas are exposed to full puff-and-pump radiating divertor conditions.

\section{Experimental setup}

The DND shape used in this study is shown in Fig. 1(a). The experimental setup is characterized by: (1) slight magnetic bias toward the lower divertor, i.e., dRsep $=-5 \mathrm{~mm}$, (2) the ion $\boldsymbol{B} \times \nabla B$ drift direction toward the upper divertor, (3) active particle exhaust by two cryo-pumps located in the upper and the one cryo-pump in lower divertor, (4) neon gas and deuterium gas are injected, respectively, into the private flux region of the lower divertor and upstream into the main chamber,

and $(5) \beta_{\mathrm{N}}(\approx 3)$ is held constant by a combination of neutral beam under feedback control and applied electron cyclotron heating $(\mathrm{ECH})$ that is held constant. 
The SF-DN used in this study [Fig. 1(b)] has the lower (primary) divertor in the Snowflakeminus configuration, while the upper (secondary) divertor is similar to that of the DND shape. Otherwise, the experimental setup and approach are identical to those of the DND (above). This facilitates subsequent comparison between SF-DN with DND performance. While the extra null in the SF-DN produces markedly different geometry along the lower outer divertor leg, both SF-DN and DND shapes have a very similar inner divertor shape.

Figures 1(c) and 1(d) represent two identically shaped core plasmas but having different isolation from their respective lower outer divertor targets. The vertical distance from their X-points to their outer divertor target were $75 \mathrm{~cm}$ and $25 \mathrm{~cm}$, respectively. These poloidal distances correspond to parallel connection lengths measured from the X-point down to the outer divertor target $\left(L_{\mid \vdash \mathrm{XPT}}\right.$ of $26 \mathrm{~m}$ and $18 \mathrm{~m}$, respectively). The poloidal distances from the X-point to the vertical inner divertor target for these cases were approximately $0.15 \mathrm{~m}$, which would correspond to parallel connection lengths of 6-8 $\mathrm{m}$. When discussing $L_{\mid \vdash \mathrm{XPT}}$ in this paper, we are referencing to the flux surface that is $1.5 \mathrm{~mm}$ radially outboard of the outer midplane separatrix flux surface.

The heat flux across the lower divertor was calculated from infrared camera measurements. The peak heat flux in the upper outer divertor was determined from measurements by a second infrared camera. Electron density and temperature at the divertor targets were determined from Langmuir probe measurements. These measurements were taken between type-1 ELMs.

\section{Results}

\subsection{High performance DND plasmas}

The response of high performance DND plasmas to the radiating divertor (RD) environment is shown in Table 1 . The RD conditions were produced by a combination of neon injection $\left(\Gamma_{\mathrm{NEON}}=\right.$ 
5.3 Torr $1 / \mathrm{s})$ and deuterium gas $\left(\Gamma_{\mathrm{D} 2}=55\right.$ Torr $\left.1 / \mathrm{s}\right)$.

To ensure that $\beta_{\mathrm{N}}$ remained constant as radiating divertor conditions were applied, power input ( $P_{\text {INPUT }}$ ) was increased, but the normalized energy confinement time $H_{98(\mathrm{y}, 2)}$ was largely unchanged. Peak heat flux at the inner target $\left(q_{\perp, \mathrm{LOW}, \mathrm{IN}}\right)$ and at the outer target $\left(q_{\perp, \mathrm{LOW}, \mathrm{OUT}}\right)$ of the lower divertor decreased by $\approx 85 \%$ and $\approx 50 \%$, respectively, as did the corresponding peak electron temperatures at the lower inner $\left(T_{\mathrm{P}, \mathrm{LOW}, \mathrm{IN}}\right)$ and lower outer $\left(T_{\mathrm{P}, \mathrm{LOW}, \mathrm{OUT}}\right)$ divertor targets. Greater heat flux reduction at the inner target than at the outer target may, in part, be due to the fact that there is no active particle pumping at the inner target; hence impurities can build up (and radiate) at the inner target more so than at the outer target, which has active particle pumping. In addition, the peak heat flux at the upper outer divertor target $\left(q_{\perp, \mathrm{UP}, \mathrm{OUT}}\right)$ increased by $\approx 20 \%$ after the RD was applied; likewise, the peak electron temperature in the upper outer divertor ( $\left.T_{\mathrm{P}, \mathrm{UP}, \mathrm{OUT}}\right)$ also increased slightly. These results may be consequences of imposing the constraint that $\beta_{\mathrm{N}}$ stay constant and of the increase in the radiated power primarily being from the lower divertor.

Total radiated power $\left(P_{\mathrm{RAD}, \mathrm{TOT}}\right)$ increased strongly after the RD was applied. Approximately $60 \%$ of $P_{\text {INPUT }}$ was radiated during the RD phase, but only $\approx 20 \%$ of $P_{\text {INPUT }}$ was radiated inside the main plasma. About half of the increase in the total radiated power occurred in the lower (primary) divertor, while $\approx 30 \%$ and $\approx 20 \%$ of this increase came from the main plasma and upper (secondary) divertor, respectively. Power balance measurements indicate that more power flowed into the scrape-off layer in the RD phase, but this "extra" power flowing to the lower divertor was more than offset by a strong increase in localized radiated power in the lower divertor. The increase in power flowing to the upper outer divertor after the $\mathrm{RD}$ was applied was more in-line with the increase in localized radiated power in the upper divertor, so that $q_{\perp, \mathrm{UP}, \mathrm{OUT}}$ (and $T_{\mathrm{P}, \mathrm{UP}, \mathrm{OUT}}$ ) did 
not show significant change. More detailed modeling efforts are underway.

While carbon from the graphite tiles was the dominant contributor to the plasma $Z_{\mathrm{eff}}$ in the nonradiating divertor case, the nearly factor of two increase in $Z_{\text {eff }}$ during $\mathrm{RD}$ originated almost entirely from the neon. The carbon concentration in the main plasma showed little change between radiating and non-radiating cases and was typical for the DND plasmas in this study. For example, Fig. 2 shows neon concentration at $\rho=0.7$ in the main plasma as a function of the neon injection rate $\Gamma_{\mathrm{NEON}}$ for two different $\Gamma_{\mathrm{D} 2}$. Neon content of the main plasma increased with $\Gamma_{\mathrm{NEON}}$ but at a slower rate than at the higher $\Gamma_{\mathrm{D} 2}$. This behavior is consistent with previous results for more conventional ELMing H-mode plasmas [4]. There is no evidence that carbon sputtering changed significantly during these scans, given that the carbon content of the main plasma remained in a narrow band regardless of $\Gamma_{\mathrm{NEON}}$ and $\Gamma_{\mathrm{D} 2}$. Since the increase in net power through the pedestal after the RD was applied was modest, the frequency of the edge localized modes (ELM) $v_{E L M}$ was only slightly higher than that of the non-RD case (Table 1).

High performance DND plasmas in DIII-D frequently run with a minimum value of their safety factor $q_{\mathrm{MIN}}$ well above unity in order to take advantage of the expected improvements in energy confinement and $\beta$ limits [13]. For the strongly radiating divertor in this study, maintaining a steady elevated $q_{\mathrm{MIN}}$ was not achieved. Whether this was the result of the inherent cooling in the plasma pedestal region (and consequential current profile peaking) or simply unoptimized operation is an open question.

\subsection{High performance $S F-D N$ plasmas}

High performance SF-DN plasmas largely mirrored the high performance characteristics of DNDs under both radiating- and non-radiating divertor conditions. This is not unexpected, because 
these SF-DN and DND plasmas have very similar poloidal cross-sections, upper and lower triangularities, and $q_{95}(=5.0-5.2)$.

Because of their nearly identical lower inner divertor geometry and poloidal flux expansion (气6.6), both SF-DN and DND heat flux profiles at their respective inner divertor targets were similar, both prior to and during the radiating phases [Fig. 3(a,c)]. On the other hand, the advantage of the SF-DN lies in a much larger poloidal flux expansion at its lower outer divertor target, i.e., $F_{\mathrm{EXP}-\mathrm{OD}} \cong 28$ for the $\mathrm{SF}-\mathrm{DN}$ versus $\cong 9$ for the DND; this resulted in nearly a $50 \%$ lower peak heat flux at the outer divertor target with the SF-DN shape, both before and during puff-and-pump operation [Fig. 3(b,d)]. One notes that $q_{\perp, \mathrm{p}}$ at the lower outer divertor target for the SF-DN in the non-radiating divertor case was still lower than $q_{\perp, \mathrm{p}}$ for the corresponding DND in the radiating divertor case.

Neon accumulation in the SF-DN, however, was 30\%-40\% higher than comparable DND cases with similar $\Gamma_{\mathrm{D} 2}$ and $\Gamma_{\mathrm{NEON}}$, and in general the line-averaged density also tended to be higher in the SF-DN cases. We hypothesize that is partly attributable to the relative difficulty in pumping the SF$\mathrm{DN}$ at the outer divertor target because of its large poloidal flux expansion. Hence, an improved understanding of how best to pump a SF-DN configuration is an important next step in assessing its application to future tokamak devices.

\subsection{Parallel connection length}

Significantly reduced $q_{\perp, \mathrm{p}}$ fin the long $L_{\mid \vdash \mathrm{XPT}}(=26 \mathrm{~m})$ case was maintained over the comparably-prepared short $L_{\mid \vdash \mathrm{XPT}}(=18 \mathrm{~m})$ case for the wide range in operating conditions studied [Fig. 4(a,b)]. Except at the lowest values of $\Gamma_{\mathrm{D} 2}$ in Fig. 4(a), the outer strike points were partially detached. All plasmas in Fig. 4(b) were partially detached, and in the $26 \mathrm{~m}$ case at highest $\Gamma_{\mathrm{NEON}}$, 
the IR camera and Langmuir probe measurements under the outer divertor leg suggest a nearcomplete detachment during between-ELM intervals.

Table 2 compares a long $L_{\mid-\mathrm{XPT}}$ case with its short $L_{\mid \vdash \mathrm{XPT}}$ case counterpart under both nonradiating (NO-RD) and strong radiating divertor (RD) conditions. The longer $L_{\mid \vdash \mathrm{XPT}}$ case had lower $q_{\perp, \mathrm{p}}$ outer target in both NO-RD and RD cases, even though its poloidal flux expansion $F_{\mathrm{EXP}-\mathrm{OD}}$ was less than that of the shorter $L_{\mid \vdash \mathrm{XPT}}$ case. Normalized energy confinement $H_{89 \mathrm{P}}$, Greenwald fraction $n_{\mathrm{e}} / n_{\mathrm{G}}$, and $Z_{\mathrm{eff}}$ were comparable for long and short $L_{\mid \vdash \mathrm{XPT}}$.

\section{Summary}

The experiments described here are the first steps toward evaluating solutions to the high power loading expected in future generation high-powered tokamaks. Thus far, we have documented attractive features in each of the three-divertor concepts operating under radiating divertor conditions (e.g., good heat flux reduction and energy confinement). Interestingly, we have also noted that the beneficial effect of a radiating divertor may not be as strong when applied to a configuration with already favorable target heat loads, as, for example, observed in the SF-DN. Possible downsides were also identified. For example, impurity contamination of the core plasma (and resulting "fuel dilution") can be an important drawback, e.g., $Z_{\text {eff }}$ for both DND and SF-DN plasmas was well above 3 during RD operation at the higher levels of $P_{\text {INPUT }}$ used in these studies. More ominously, core contamination could present a more difficult challenge with SF-DN, because its higher $F_{\text {EXP-OD }}$ make particle pumping (and impurity removal) more difficult. Future experiments should address this issue.

Increasing $L_{\mid \vdash \mathrm{XPT}}$ reduced $q_{\perp, \mathrm{p}}$ at the outer divertor target under both $\mathrm{RD}$ and non-RD conditions. We also found that plasmas with shorter $L_{\mid \vdash \text { XPT }}$ had higher core density under similar 
$\Gamma_{\mathrm{D} 2}$. This was likely due to higher $F_{\mathrm{EXP}-\mathrm{OT}}$ at the outer target for the short $L_{\mid-\mathrm{XPT}}$ case, which in turn reduced pumping effectiveness (similar to SF-DN cases). However, this problem might be resolvable by better control over poloidal flux expansion near the pumping ports.

\section{Acknowledgment}

This material is based upon work supported by the U.S. Department of Energy, Office of Science, Office of Fusion Energy Sciences, using the DIII-D National Fusion Facility, a DOE Office

of Science user facility, under Award DE-FC02-04ER54698, DE-AC52-07NA27344, DE-AC0209CH11466, DE-FG02-07ER54917, DE-FG02-04ER54541, and DE-AC04-94AL85000. DIII-D data shown in this paper can be obtained in digital format by following the links at https://fusion.gat.com/global/D3D_DMP.

\section{References}

[1] M.R. Wade et al., Nucl. Fusion 38 (1998) 1839.

[2] J.A. Goetz et al., J. Nucl. Mater. 266-269 (1999) 359.

[3] A. Kallenbach et al., J. Nucl. Mater. 337-339 (2005) 732.

[4] T.W. Petrie et al., J. Nucl. Mater. 363-365 (2007) 416.

[5] D.D. Ryutov, Phys. Plasmas 14 (2007) 064502.

[6] M. Kotschenreuther, Phys. Plasmas 14 (2007) 072502.

[7] T.H. Osborne et al., Plasma Phys. Control. Fusion 42 (2000) A175.

[8] J.R. Ferron et al., Phys. Plasmas 12 (2005) 056126

[9] V.A. Soukhanovskii et al., Phys. Plasmas 19 (2012) 082504. 
[10] D.N. Hill et al., Nucl. Fusion 53 (2013) 104001.

[11] T.W. Petrie et al., Nucl. Fusion 53 (2013) 113024.

[12] R. Schneider et al., Contrib. Plasma Phys. 46, 3 (2006).

[13] A.D. Turnbull et al., Phys. Rev. Lett. 74 (1995) 718. 
Table 1: High performance DND plasmas with and without radiating divertor

\begin{tabular}{|l|l|l|}
\hline & No RD & RD \\
\hline$\beta_{\mathrm{N}}$ & 2.9 & 2.9 \\
\hline $\mathrm{H}_{98(\mathrm{Y}, 2)}$ & 1.35 & 1.30 \\
\hline $\mathrm{n}_{\mathrm{e}} / \mathrm{n}_{\mathrm{GREENWALD}}$ & 0.41 & 0.56 \\
\hline $\mathrm{P}_{\text {INPUT }}(\mathrm{MW})$ & 8.8 & 10.0 \\
\hline $\mathrm{P}_{\mathrm{RAD}, \mathrm{TOT}}(\mathrm{MW})$ & 3.1 & 6.0 \\
\hline $\mathrm{P}_{\mathrm{RAD}, \mathrm{CORE}}(\mathrm{MW})$ & 1.0 & 1.9 \\
\hline $\mathrm{q}_{\perp, \mathrm{LOW}, \mathrm{OUT}}$ & 3.20 & 1.70 \\
$\left.\mathrm{MMW}^{2}\right)$ & & \\
\hline $\mathrm{q}_{\perp, \mathrm{LOW}, \mathrm{IN}}\left(\mathrm{MW} / \mathrm{m}^{2}\right)$ & 1.25 & 0.19 \\
\hline $\mathrm{q}_{\perp, \mathrm{UP}, \mathrm{OUT}}\left(\mathrm{MW} / \mathrm{m}^{2}\right)$ & 1.20 & 1.35 \\
\hline $\mathrm{T}_{\mathrm{P}, \mathrm{LOW}, \mathrm{OUT}}(\mathrm{eV})$ & 55 & 18 \\
\hline $\mathrm{T}_{\mathrm{P}, \mathrm{LOW}, \mathrm{IN}}(\mathrm{eV})$ & 40 & 15 \\
\hline $\mathrm{T}_{\mathrm{P}, \mathrm{UP}, \mathrm{OUT}}(\mathrm{eV})$ & 14 & 15 \\
\hline $\mathrm{v}_{\mathrm{ELM}}(\mathrm{Hz})$ & 80 & 96 \\
\hline$Z_{\mathrm{eff}}$ & 1.7 & 3.4 \\
\hline $\mathrm{q}_{\mathrm{MIN}}$ & 1.5 & 1.1 \\
\hline
\end{tabular}


Table 2: Long vs short parallel connection length cases with and without strong radiating conditions

\begin{tabular}{|l|c|c|c|c|}
\hline & \multicolumn{2}{|c|}{ High X-point } & \multicolumn{2}{c|}{ Low X-point } \\
\hline & No RD & RD & No RD & RD \\
\hline $\mathrm{L}_{\|, \mathrm{XPT}}(\mathrm{m})$ & 26 & 26 & 18 & 18 \\
\hline$\beta_{\mathrm{N}}$ & 1.9 & 1.9 & 1.9 & 1.0 \\
\hline $\mathrm{F}_{\text {EXP-OD }}$ & 2.7 & 2.7 & 4.2 & 4.2 \\
\hline$\Gamma_{\mathrm{D} 2}($ Torr $1 / \mathrm{s})$ & 30 & 150 & 6 & 150 \\
\hline$\Gamma_{\text {NEON }}($ Torr $1 / \mathrm{s})$ & 0.4 & 5.3 & 0.4 & 5.3 \\
\hline $\mathrm{P}_{\mathrm{INPUT}}(\mathrm{MW})$ & 6.4 & 9.2 & 6.6 & 8.9 \\
\hline $\mathrm{q}_{\perp, \mathrm{P}}\left(\mathrm{MW} / \mathrm{m}^{2}\right)$ & 1.5 & 0.4 & 2.6 & 0.8 \\
\hline $\mathrm{H}_{89 \mathrm{P}}$ & 1.55 & 1.30 & 1.55 & 1.30 \\
\hline $\mathrm{n}_{\mathrm{e}} / \mathrm{n}_{\mathrm{G}}$ & 0.35 & 0.54 & 0.36 & 0.56 \\
\hline$Z_{\mathrm{eff}}$ & 1.44 & 1.84 & 1.30 & 1.86 \\
\hline
\end{tabular}




\section{Figure Captions}

Fig. 1. The plasma cross-sections for the four H-mode plasmas discussed in this paper are shown. Plasma performance under RD evaluated for: (a) DND that is magnetically biased toward the lower (primary) divertor $(\mathrm{dRsep}=-0.5 \mathrm{~cm}),(\mathrm{b})$ double-null with "Snowflake" lower divertor and upper (secondary) divertor similar to the DND in (a), (c) longer outer divertor leg $\left(L_{\mid \vdash \mathrm{XPT}}=26 \mathrm{~m}\right)$ and $(\mathrm{d})$ the corresponding shorter outer divertor leg $\left(L_{\mid-\mathrm{XPT}}=18 \mathrm{~m}\right)$.

Fig. 2. Neon density at $\rho=0.7$ inside the main plasma as a function of the neon injection rate

$\Gamma_{\mathrm{NEON}}$. The range in the carbon density in the scan at $\rho=0.7$ is also shown. Parameters: $q_{95}=4.7$, $H_{98(\mathrm{y}, 2)}=1.25-1.40, P_{\mathrm{INPUT}}=9-11 \mathrm{MW}$ and $n_{\mathrm{e}} / n_{\mathrm{G}}=0.45-0.55$.

Fig. 3. Heat flux profiles of DND and corresponding SF-DN during both non-puff (red) and full radiating divertor (blue) cases; Inner and outer divertor target of the DND (a,b); and SF-DN (c,d). Parameters: $q_{95}=5.2, H_{98(\mathrm{y}, 2)}=1.2-1.4, P_{\mathrm{IN}}=10 \mathrm{MW}$, and $n_{\mathrm{e}} / n_{\mathrm{G}}=0.45-0.60$.

Fig. 4. (a) Peak heat flux at the outer divertor target versus $\Gamma_{\mathrm{D} 2}$ for $L_{\mid \vdash \mathrm{XPT}}=18 \mathrm{~m}$ and $26 \mathrm{~m}$ : $\beta_{\mathrm{N}}=1.9, \mathrm{P}_{\mathrm{INPUT}}=7-9 \mathrm{MW}$, and trace neon. (b) Peak heat flux at the outer divertor target versus $\Gamma_{\mathrm{NEON}}$ for the same values of $L_{\mid-\mathrm{XPT}}: \beta_{\mathrm{N}}=1.9, P_{\mathrm{INPUT}}=9 \mathrm{MW}$, and $\Gamma_{\mathrm{D} 2}=150$ Torr $1 / \mathrm{s}$. 


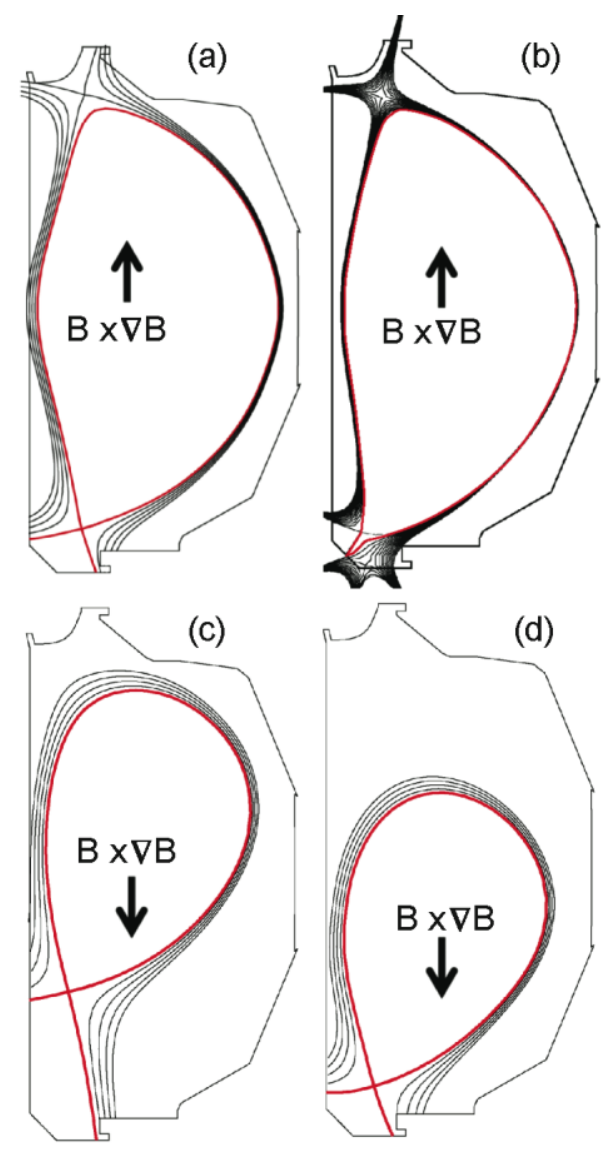

Figure $1 \quad(111 \mathrm{~mm})$ 


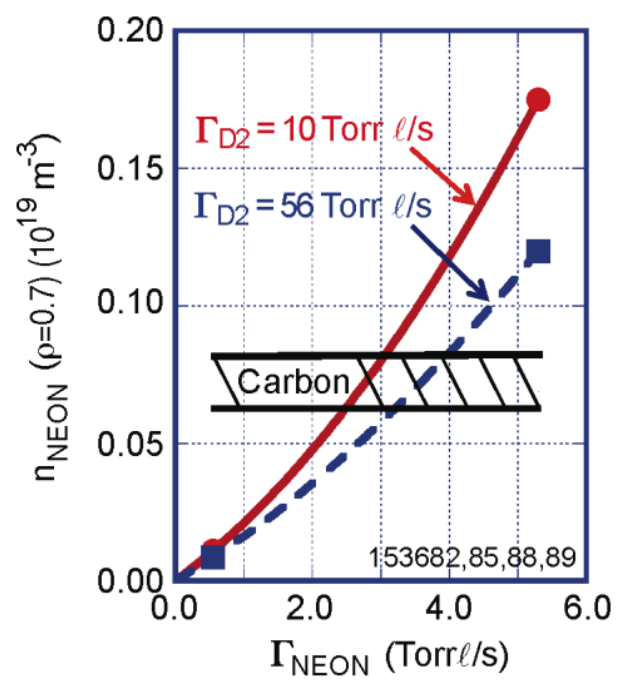

Figure $2 \quad(68 \mathrm{~mm})$ 


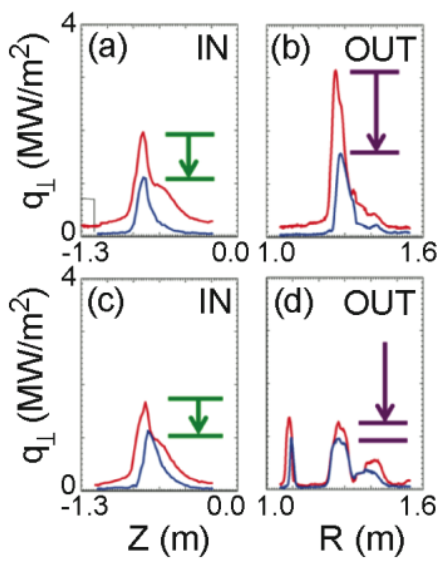

Figure $3 \quad(56 \mathrm{~mm})$
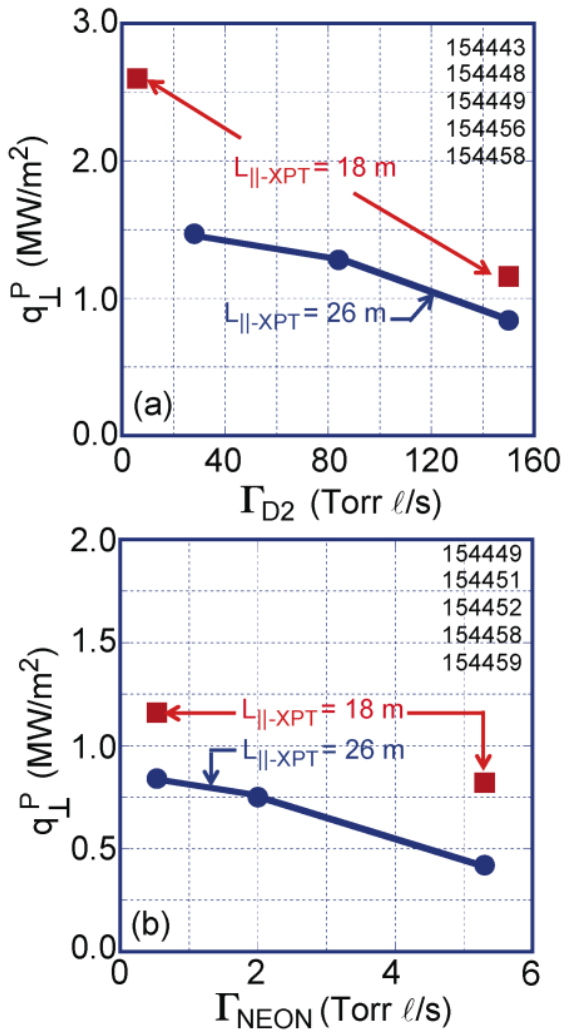

Figure $4 \quad(108 \mathrm{~mm})$ 